\title{
Autosomal recessive bestrophinopathy associated with angle-closure glaucoma
}

\author{
C. Crowley • R. Paterson • T. Lamey • \\ T. McLaren · J. De Roach $\cdot$ E. Chelva • \\ J. Khan
}

Received: 16 December 2013/Accepted: 12 May 2014/Published online: 24 May 2014

(C) The Author(s) 2014. This article is published with open access at Springerlink.com

\begin{abstract}
Purpose Abnormalities in the BEST1 gene have recently been recognised as causing autosomal recessive bestrophinopathy (ARB). ARB has been noted to have a variable phenotypic presentation, distinct from that of autosomal dominant Best vitelliform macular dystrophy (BVMD). Both conditions are associated with deposits in the retina, a reduced or absent electro-oculography (EOG) light rise, and the risk of developing angleclosure glaucoma. Herein, we describe the clinical and genetic characteristics of a young male diagnosed with $\mathrm{ARB}$ associated with angle-closure glaucoma resulting from a novel homozygous mutation in BEST1.

Methods All research involved in this case adhered to the tenets of the Declaration of Helsinki. The proband underwent slitlamp examination, retinal autofluorescence imaging and optical coherence tomography after presenting with deteriorating vision. The findings prompted genetic testing with bi-directional DNA sequencing of coding and flanking intronic regions of BEST1. The proband's family members were subsequently screened.
\end{abstract}

C. Crowley $(\bowtie) \cdot$ R. Paterson - T. Lamey ·

T. McLaren · J. De Roach · E. Chelva · J. Khan Department of Medical Technology and Physics, Sir Charles Gairdner Hospital, Hospital Avenue, Nedlands, WA 6009, Australia

e-mail: claire.crowley86@gmail.com

T. Lamey $\cdot$ J. Khan

Centre for Ophthalmology and Visual Science, University of Western Australia, Nedlands, WA 6009, Australia
Results A provisional diagnosis of ARB was made based on the findings of subretinal and schitic lesions on fundoscopy and retinal imaging, together with abnormal EOG and electroretinography. Genetic testing identified a novel homozygous mutation in BEST1, c.636+1 G $>$ A. Family members were found to carry one copy of the mutation and had no clinical or electrophysiological evidence of disease. The proband was additionally diagnosed with angle-closure glaucoma requiring topical therapy, peripheral iridotomies and phacoemulsification.

Conclusions Phenotypic overlap, reduced penetrance, variable expressivity and the ongoing discovery of new forms of bestrophinopathies add to the difficulty in distinguishing these retinal diseases. All patients diagnosed with ARB or BVMD should be examined for narrow angles and glaucoma, given their frequent association with these conditions.

Keywords BESTl gene - Autosomal recessive bestrophinopathy $\cdot$ Best vitelliform macular dystrophy · Angle-closure glaucoma ·

Electroretinography · Electro-oculography · Intraocular pressure

\section{Introduction}

Autosomal recessive bestrophinopathy (ARB) has recently been described and has a more global influence on eye development and physiology than autosomal 
dominant Best vitelliform macular dystrophy (BVMD), otherwise known as Best disease. We present a case of ARB associated with narrow-angle glaucoma and a novel homozygous mutation in BEST1.

\section{Case description}

A 26-year-old male presented with deteriorating vision in both eyes. He had no family history of eye disorders but had been diagnosed with possible Stargardt disease at the age of 12 years. This diagnosis was amended to exudative polymorphous vitelliform macular dystrophy two years later when he underwent electrophysiological studies which revealed normal rod electroretinography (ERG) but abnormal flicker ERG with b-wave delay and abnormal electro-oculography (EOG) (Fig. 1a). A trial of steroids had no benefit at that time. a
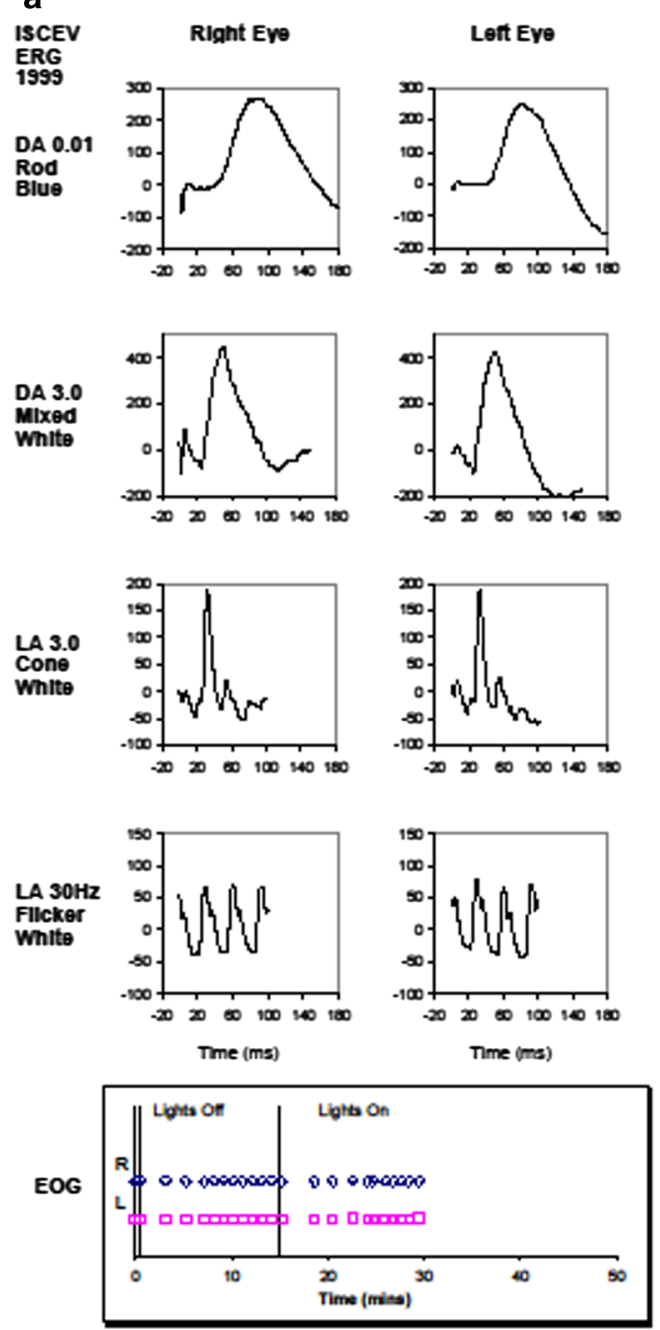

EOG

Fig. 1 a 1999 electrophysiology which showed normal darkadapted ERG but abnormal flicker ERG with b-wave delay and grossly abnormal EOG. Recordings were made on a custom built electrophysiology system according to current ISCEV standards at the time. The ERG was measured with Burian-Allen corneal contact lens electrodes. b 2011 electrophysiology which

b

LA 3.0

$\mathrm{LA} \mathrm{30H2}$
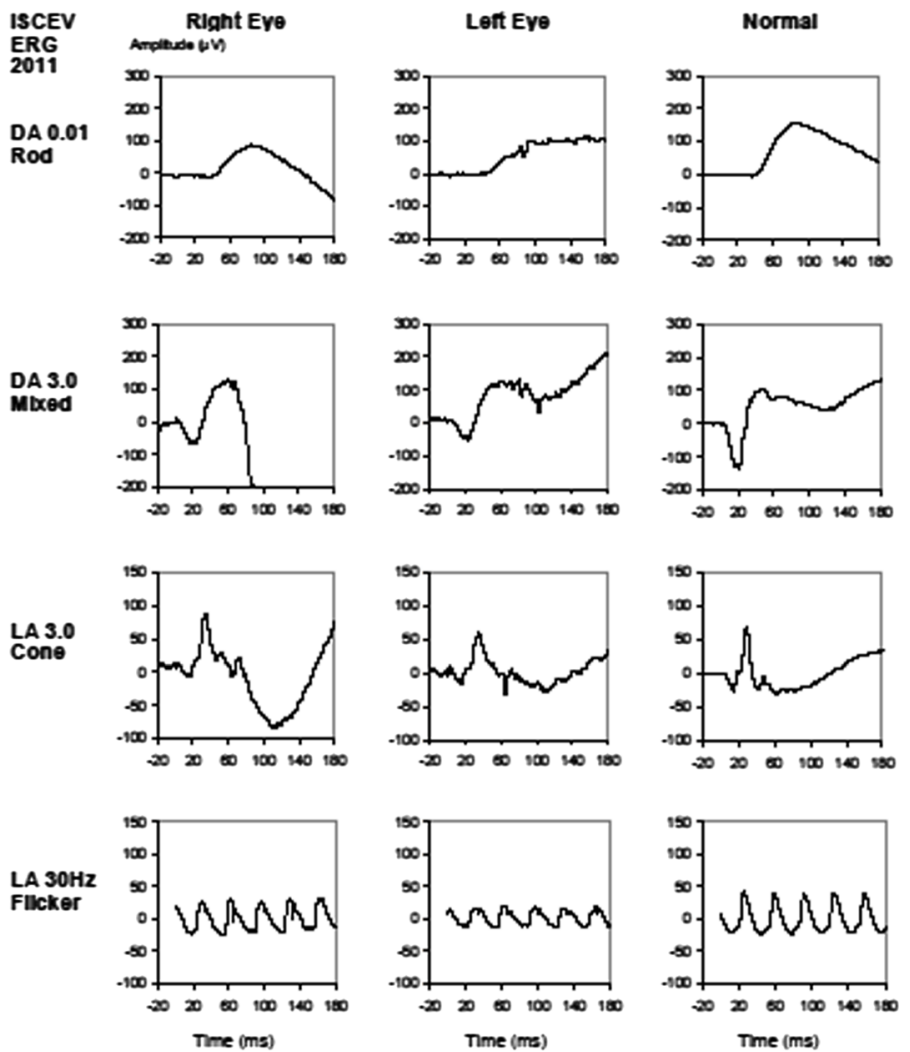

nme (ms)

Thme (me)

Trme (ms)

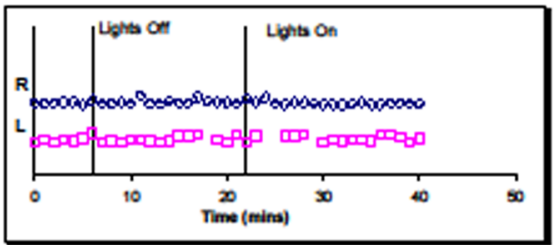

showed deterioration in the dark-adapted ERG with delayed and reduced responses, whilst light-adapted cone and flicker ERG were further delayed. The EOG remained grossly abnormal. Recordings were made on the LKC EMWIN E-3000 System according to current ISCEV standards. The ERG was measured with HK-Loop electrodes 
He was referred to us for a second opinion 12 years later. Best-corrected visual acuity (BCVA) was 6/36 right and 6/60 left. He had minimal refractive error of 0.5 dioptre spherical equivalent hyperopia in each eye. Intraocular pressure (IOP) was $13 \mathrm{mmHg}$ right and $16 \mathrm{mmHg}$ left. Fundoscopy revealed normal optic discs but a grossly abnormal retina bilaterally, with widespread subretinal yellow lesions which autofluoresced (Fig. 2a) and schitic changes at the foveae (Fig. 2b). Electrophysiology had deteriorated significantly and now demonstrated delayed and reduced rod responses, further delayed photopic and flicker ERG and reduced P50 in the pattern ERG and a grossly abnormal multifocal ERG. The EOG remained grossly abnormal (Fig. 1b). Testing for possible infective and inflammatory conditions was negative.
A provisional diagnosis of ARB was made, and a trial of oral acetazolamide initiated in the light of reports of improvement in schitic changes in retinal dystrophies with carbonic anhydrase inhibitors [1]. Anatomical improvement in the central retinal thickness was noted whilst on treatment over a 6-month period. BCVA improved to $6 / 18$ right eye, with no change in that for the left (Fig. 3).

The day after one of his follow-up visits during which dilated fundoscopy and retinal imaging was performed, and the dose of his acetazolamide was halved to $250 \mathrm{mg}$ twice daily due to mild systemic side effects, he returned for unscheduled review, reporting left eye discomfort and increasing floaters. Visual acuity was unchanged. However, left IOP was markedly raised at $38 \mathrm{mmHg}$, compared with $4 \mathrm{mmHg}$ for

\section{a}
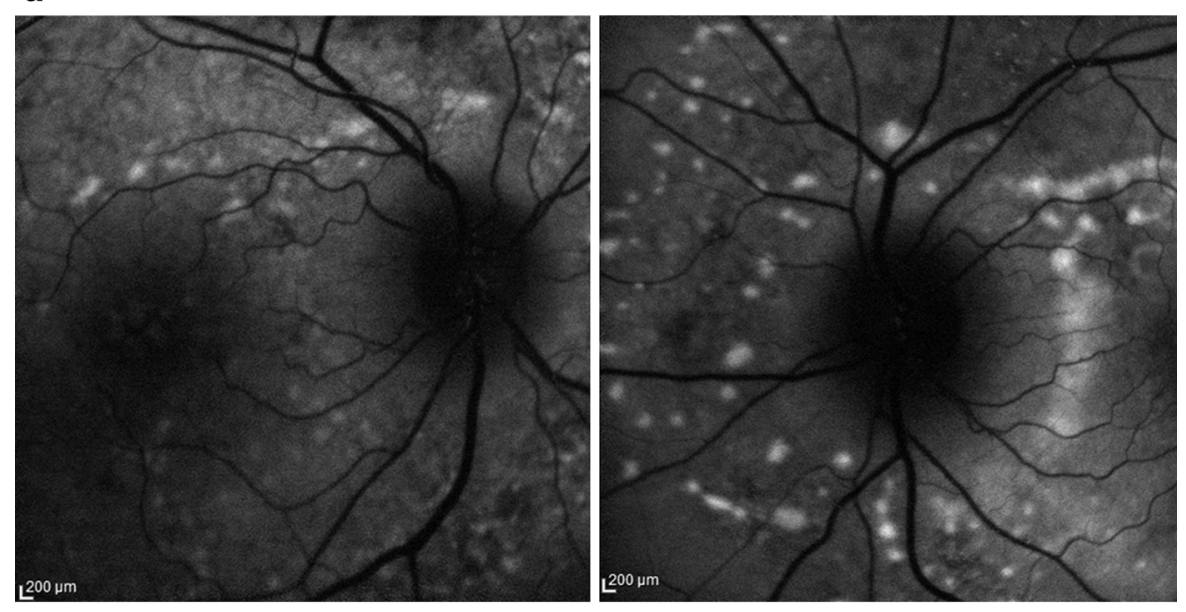

b

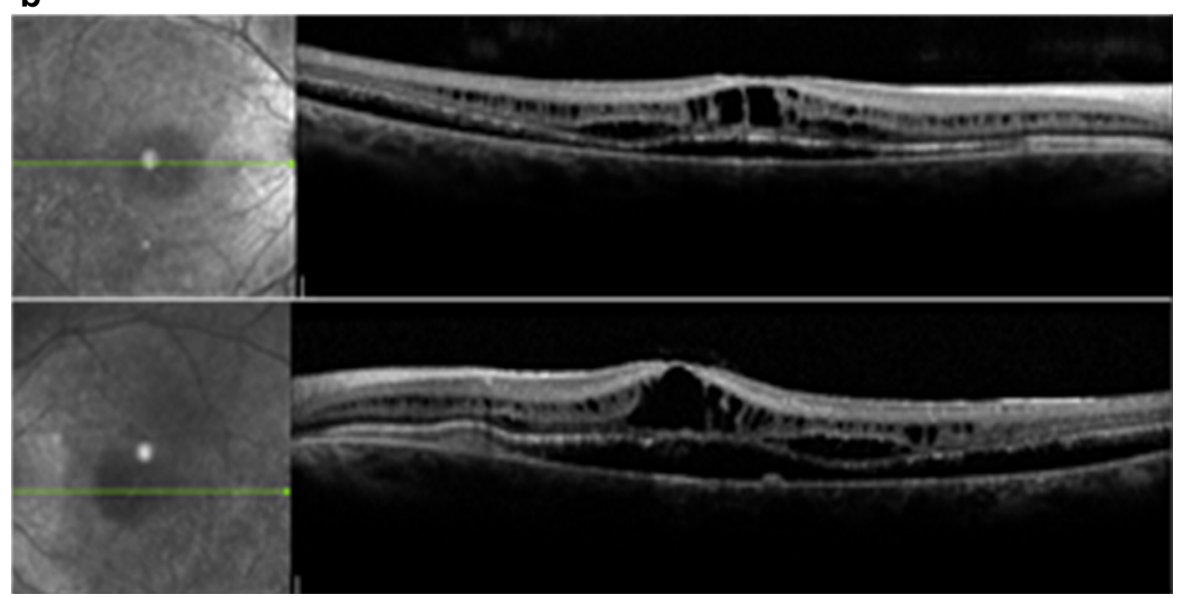

Fig. 2 a Right and left autofluorescent images demonstrating widespread autofluorescent lesions typical for ARB. b Right and left spectral domain OCT images demonstrating extensive retinoschisis, intraretinal and subretinal fluid 

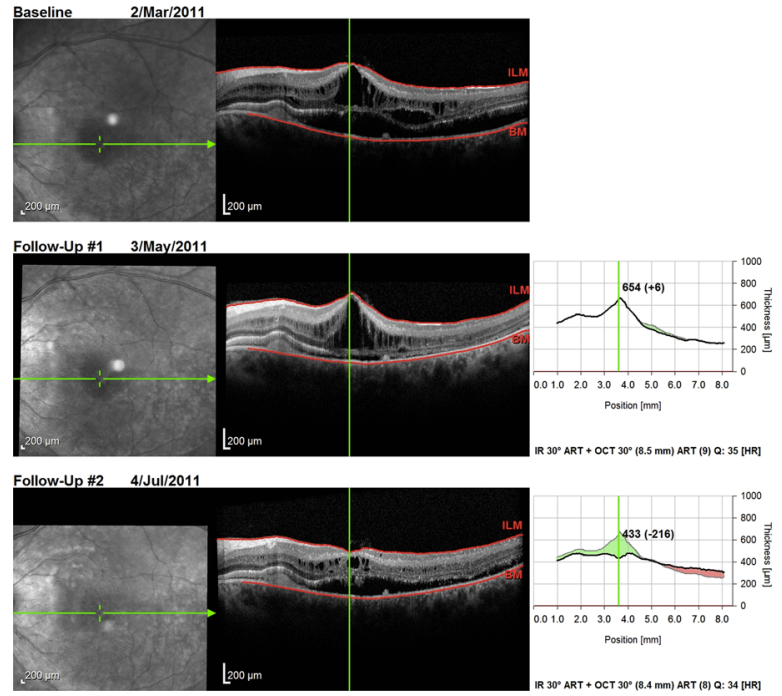
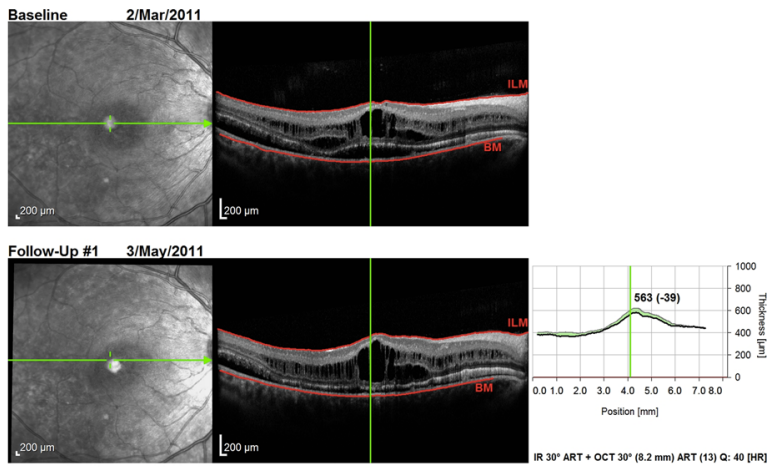

Follow-Up\#2 4/Jul/2011

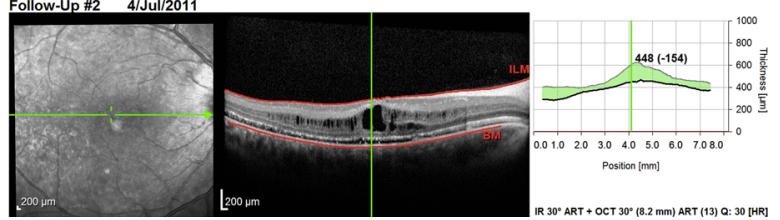

Fig. 3 Right and left spectral domain OCT images demonstrating redistribution of intraretinal and subretinal fluid after 4 months of oral acetazolamide

his right eye. A narrow drainage angle was noted bilaterally, but it was significantly narrower in the left eye, with a shallow anterior chamber and 'volcano sign'. Subacute angle-closure glaucoma was diagnosed and effectively treated initially with topical antihypertensives and peripheral iridotomies. However, left-sided IOP continued to rise over a few days to $60 \mathrm{mmHg}$. B-scan ultrasound excluded choroidal effusion and confirmed plateau iris. His axial length measured $21.64 \mathrm{~mm}$ right and $22.06 \mathrm{~mm}$ left, and his anterior chamber depth was $2.48 \mathrm{~mm}$ right and $2.47 \mathrm{~mm}$ left. His optic disc was not cupped, with a cup-to-disc ratio of less than 0.4 bilaterally. Phacoemulsification lens extraction was eventually required to lower the IOP, and a 24.0 dioptre posterior chamber lens (A-constant 119.0) was inserted uneventfully. IOP was controlled at $18 \mathrm{mmHg}$ thereafter but required continued use of topical prostaglandin and beta-blocker.

Bi-directional DNA sequencing of coding and flanking intronic regions of BEST1 revealed that the patient was homozygous for a novel splice variant, c. $636+1 \mathrm{G}>$ A. This substitution was predicted to be pathogenic by abolishing the conserved donor splice site (Mutation Taster; Human Splicing Finder v2.4.1; NN SPLICE) [2], with potential usage of a cryptic donor splice site present 294 bp downstream in intron 5 (0.88 NN SPLICE). Cascade family testing identified this patient's non-consanguineous parents and sister as carriers. They were asymptomatic and did not show signs of disease, with completely normal vision, fundi, anterior segments and electrophysiology.

\section{Discussion}

The BEST1 gene, formally known as VMD2, encodes bestrophin-1, previously postulated to act as a $\mathrm{Ca}^{2+}$. activated chloride channel [3], a regulator of voltagegated $\mathrm{Ca}^{2+}$ channels [4], or a $\mathrm{HCO}_{3}{ }^{-}$channel [5] in the basolateral membrane of the RPE [6]. It was recently shown to localise in the endoplasmic reticulum membrane, however [7]. Bestrophin-1 dysfunction has been associated with defective regulation of subretinal fluid reabsorption and aberrant phagocytosis of the photoreceptor discs [8]. Over 250 diseasecausing mutations have been identified in the BEST1 gene to date associated with a broad range of phenotypes, including BVMD, adult vitelliform macular dystrophy, autosomal dominant vitreoretinochoroidopathy (ADVIRC), the MRCS (microcornea, rodcone dystrophy, cataract, posterior staphyloma) syndrome, retinitis pigmentosa and ARB [9-14].

ARB is thought to result from biallelic functionally null mutations of the gene, whilst most dominantly inherited missense mutations have been found to produce dominant negative effects and so do not compromise protein synthesis [10, 12, 13]. In vitro 
studies using HEK293 cells showed that co-transfection of the two mutations observed in the compound heterozygous state in ARB abolished chloride conductance in contrast to co-transfection of a single mutant with wild-type bestrophin-1 which led to significantly smaller chloride currents compared to wild-type bestrophin-1 $[9,15]$. This suggests that the autosomal recessive phenotype only manifests when bestrophin-1 activity falls below a functional threshold. Davidson et al. [15] also found that different ARB-associated mutants lead to the same disease phenotype but through different effects on cellular processing mechanisms. This finding has implications for potential gene replacement therapies as the authors showed that missense mutations associated with autosomal recessive diseases may have a pathogenic outcome beyond simple loss of function.

Whilst BVMD is characterised by vitelliform lesions that typically occur at the macula as a result of abnormal deposition of lipofuscin in the retinal pigment epithelium (RPE), ARB is associated with subretinal deposits occurring predominantly outside the macula, mainly at the posterior pole and along the vascular arcades [9, 16-19]. These are often small and fleck-like or punctate in shape, white or yellow in colour, and hyperfluoresce on fundus autofluorescence imaging. Optical coherence tomography (OCT) shows subretinal and intraretinal fluid accumulation, often at the maculae [9, 18-21]. Using higher resolution Fourier-domain OCT, Gerth et al. [17] demonstrated RPE deposits and significant photoreceptor changes but preserved inner retinal layers in an 11-year-old boy with ARB, including thickened, elongated photoreceptor outer segments and detachment from the RPE.

Unlike BVMD, ARB is associated with diminished rod- and cone-driven ERG responses, though it shares the presence of a severely reduced or absent EOG light rise with BVMD as well as ADVIRC [9, 10, 16-24]. The expression of BEST1 is higher in the peripheral RPE than at the macula [25]. This may explain the more widespread and progressive photoreceptor dysfunction, as well as the predominantly peripheral location of retinal lesions observed in patients with ARB. No histopathological data are available due to the novel description of the ARB phenotype.

Since ARB was first recognised in 2008 by Burgess et al. [9], at least 35 novel compound heterozygous and homozygous mutations have been reported to cause the disorder $[9,16-19,21,26-31]$. Incomplete penetrance and variability in expression associated with some mutations in BEST1, together with the fact that some phenotypic distinctions may not develop until later years, make it difficult to distinguish those resulting in dominant inheritance from those resulting in recessive inheritance.

A family reported by Schatz et al. in 2006 [32] with compound heterozygous BEST1 mutations is thought by some to have represented ARB rather than 'atypical' BVMD [9]. However, the heterozygous carriers also had ERG and EOG abnormalities, suggesting a diagnosis of BVMD with reduced penetrance in those individuals. Sharon et al. [30] recently reported a novel BEST1 mutation in a Danish family in which the proband also had a previously reported mutation of the other allele and a phenotype suggestive of ARB. However, his mother and sister who were heterozygous for the novel mutation additionally had reduced EOG light rise which would not be expected with an autosomal recessive inheritance pattern. Pineiro-Gallego et al. [28] similarly reported on a case with a homozygous mutation causing what they considered to be ARB even though heterozygotes in the family also had abnormal EOG findings. One of the two cases of ARB reported by Pomares et al. [29] had fundus findings suggestive of ARB, but the patient had a normal EOG and no family history given he was adopted and so his novel homozygous mutation cannot be confirmed to cause ARB.

Bitner et al. [33] were of the impression that BVMD can be inherited as an autosomal recessive disease and distinguished this from ARB where the patients were homozygous for a novel mutation, had fundus and electrophysiology findings in keeping with a diagnosis of BVMD and their unaffected parents each carried one copy of the same mutation. As shown by Cascavilla et al. [26] however, it may not be until later years that the ERG becomes abnormal in cases of ARB.

In addition to small eyes, reduced axial length and hyperopia, it is increasingly recognised that bestrophinopathies are also strongly associated with anterior segment abnormalities and a high incidence of narrow-angle glaucoma $[9,10,13,14,20,22-24$, 34]. The causes behind these associations are still to be elucidated, but there is evidence to support the hypothesis that BEST1 is involved through bestrophin-1 expression in the RPE in the development of ocular structures beyond the retina [10]. Wittstrom 
et al. [22] reported that two of four patients with BVMD from one pedigree exhibited shallow anterior chambers, and all four patients had hyperopia as well as reduced axial lengths. Micropthalmos (axial length $\leq 20 \mathrm{~mm}$ ) was found in two cases, both of whom had narrow angles and one of whom developed acute closed-angle glaucoma at the age of 12 years. Low et al. [34] found that sibling carriers of probands with BVMD can also have narrow anterior chamber angles, short axial lengths and a similar risk of angle-closure glaucoma.

Angle-closure glaucoma has been shown to affect approximately $50 \%$ of those with ARB. Burgess et al. [9] found that all seven cases of ARB examined were hyperopic, and three required surgery for angleclosure glaucoma. Davidson et al. [20] described two unrelated cases of ARB, both of whom had angleclosure glaucoma contributing to their visual loss. All ten patients with ARB examined by Boon et al. [18] were hyperopic, and five had shallow anterior chambers and narrow angles for which they underwent prophylactic laser peripheral iridotomies. One of these patients with additional short axial lengths underwent phacoemulsification and intraocular lens implantation in an effort to deepen the anterior chamber and its angles for both eyes. Unlike the case we have presented, this was not enough to control her IOPs and she went on to have an iris base laser iridoplasty followed by topical anti-glaucomatous therapy. The authors postulated that cataract extraction may not sufficiently open the anterior chamber angles as some patients with ARB may have a dysgenesis of the anterior segment that additionally affects the trabecular meshwork.

Although plateau iris has not been described previously in ARB, a study by Etter et al. [35] suggests that it has a heritable component, with five of the ten patients studied with plateau iris syndrome having at least one affected first-degree family member. The authors likened this to an autosomal dominant pattern of inheritance with incomplete penetrance.

In conclusion, we report a novel BESTI splice variant, c.636+1 G>A, identified homozygously in a proband clinically diagnosed with ARB in association with plateau iris and narrow-angle glaucoma. A heterozygous $\mathrm{G}>\mathrm{C}$ change in this position has been reported before, together with known pathogenic variant c.422 $\mathrm{G}>\mathrm{A}(\mathrm{R} 141 \mathrm{H})$ for a patient clinically diagnosed with BVMD [36]. The splice variant reported in the present study is likely to abolish the conserved $3^{\prime}$ splice site of exon 5 . Family members carrying one copy of the mutation had no symptoms or signs of disease, supporting a recessive inheritance pattern; however, functional studies are required to confirm pathogenicity of the variant. We strongly recommend routine screening for narrow angles and glaucoma in ARB and BVMD given their frequent association, as well as close monitoring of IOP even after peripheral iridotomy and/or phacoemulsification have been performed.

Acknowledgments The Australian Inherited Retinal Disease Register is financially supported by Retina Australia. We gratefully acknowledge the assistance of the Western Australian DNA Bank (National Health and Medical Research Council (NHMRC) Enabling Facility) with processing of DNA samples for this study and the funding provided by an Australian NHMRC Centres of Research Excellence Grant 1023911 (2012-2016). We would also like to thank Translation of Genetic Eye Research (ToGER) for its support.

Open Access This article is distributed under the terms of the Creative Commons Attribution License which permits any use, distribution, and reproduction in any medium, provided the original author(s) and the source are credited.

\section{References}

1. Iannaccone A, Fung KH, Eyestone ME, Stone EM (2009) Treatment of adult-onset acute macular retinoschisis in enhanced-S cone syndrome with oral acetazolamide. Am J Ophthalmol 147(2):307-312

2. Reese MG, Eeckman FH, Kulp D, Haussler D (1997) Improved splice site detection in Genie. J Comput Biol 4(3):311-323

3. Sun H, Tsunenari T, Yau KW, Nathans J (2002) The vitelliform macular dystrophy protein defines a new family of chloride channels. Proc Natl Acad Sci USA 99:4008-4013

4. Rosenthal R, Bakall B, Kinnick T, Peachey N, Wimmers S, Wadelius C et al (2006) Expression of bestrophin-1, the product of the VMD2 gene, modulates voltage-dependent $\mathrm{Ca}^{2+}$ channels in retinal pigment epithelial cells. FASEB J 20:178-180

5. Qu Z, Hartzell HC (2008) Bestrophin Cl- channels are highly permeable to $\mathrm{HCO}$. Am J Physiol Cell Physiol 294:C1371-C1377

6. Marmorstein AD, Marmorstein LY, Rayborn M, Wang X, Hollyfield JG, Pretrukin K (2000) Bestrophin, the product of the Best vitelliform macular dystrophy gene (VMD2), localizes to the basolateral plasma membrane of the retinal pigment epithelium. Proc Natl Acad Sci USA 97:12758-12763

7. Gomez NM, Tamm ER, Straubeta O (2013) Role of bestrophin-1 in store-operated calcium entry in retinal pigment epithelium. Pflugers Arch 465(4):481-495 
8. Xiao Q, Hartzell HC, Yu K (2010) Bestrophins and retinopathies. Pflugers Arch 460:559-569

9. Burgess R, Millar ID, Leroy BP, Urquhart JE, Fearon IM, De Baere E et al (2008) Biallelic mutation of BEST1 causes a distinct retinopathy in humans. Am J Hum Genet 1:19-31

10. Boon CJ, Klevering BJ, Leroy BP, Hoyng CB, Keunen JE, den Hollander AI (2009) The spectrum of ocular phenotypes caused by mutations in the BEST1 gene. Prog Retin Eye Res 3:187-205

11. Davidson AE, Millar ID, Urquhart JE, Burgess-Mullan R, Shweikh Y, Parry N et al (2009) Missense mutations in a retinal pigment epithelium protein, bestrophin-1, cause retinitis pigmentosa. Am J Hum Genet 85:581-592

12. Petrukhin K, Koisti MJ, Bakall B, Li W, Xie W, Marknell T et al (1998) Identification of the gene responsible for Best macular dystrophy. Nat Genet 19:241-247

13. Yardley J, Leroy BP, Hart-Holden N, Lafaut BA, Loeys B, Messiaen LM et al (2004) Mutations of VMD2 splicing regulators cause nanophthalmos and autosomal dominant vitreochoroidopathy (ADVIRC). Invest Ophthalmol Vis Sci 45:3683-3689

14. Reddy MA, Francis PJ, Berry V et al (2003) A clinical and molecular genetic study of a rare dominantly inherited syndrome (MRCS) comprising of microcornea, rod-cone dystrophy, cataract, and posterior staphyloma. Br J Ophthalmol 87:197-202

15. Davidson AE, Miller ID, Burgess-Mullan R, Maher GJ, Urquhart JE, Brown PD et al (2011) Functional characterization of bestrophin-1 missense mutations associated with autosomal recessive bestrophinopathy. Invest Ophthalmol Vis Sci 52:3730-3736

16. Kinnick TR, Mullins RF, Dev S, Leys M, Mackey DA, Kay $\mathrm{CN}$ et al (2011) Autosomal recessive vitelliform macular dystrophy in a large cohort of vitelliform macular dystrophy patients. Retina 3:581-595

17. Gerth C, Zawadzki RJ, Werner JS, Heon E (2009) Detailed analysis of retinal function and morphology in a patient with autosomal recessive bestrophinopathy (ARB). Doc Ophthalmol 3:239-246

18. Boon CJ, van der Born LI, Visser L, Keunen JE, Bergen B, Booij JC et al (2013) Autosomal recessive bestrophinopathy. Differential diagnosis and treatment options. Ophthalmology 120:809-820

19. Borman AD, Davidson AE, O' Sullivan J, Thompson DA, Robson AG, De Baere E et al (2011) Childhood-onset autosomal recessive bestrophinopathy. Arch Ophthalmol 129(8):1088-1093

20. Davidson AE, Sergouniotis PI, Burgess-Mullan R, HartHolden N, Low S, Foster PJ et al (2010) A synonymous codon variant in two patients with autosomal recessive bestrophinopathy alters in vitro splicing of BEST1. Mol Vis 16:2916-2922

21. Iannacone A, Kerr NC, Kinnick TR, Calzada JI, Stone EM (2011) Autosomal recessive best vitelliform macular dystrophy: report of a family and management of early-onset neovascular complications. Arch Ophthalmol 2:211-217

22. Wittström E, Ponjavic V, Bondeson ML, Andréasson S (2011) Anterior segment abnormalities and angle-closure glaucoma in a family with a mutation in the BEST1 gene and best vitelliform macular dystrophy. Ophthalmic Genet 32(4):217-227
23. Bard LA, Cross HE (1975) Genetic counseling of families with Best macular dystrophy. Trans Sect Ophthalmol Am Acad Ophthalmol Otolaryngol 79(6):OP865-OP873

24. Sohn EH, Francis PJ, Duncan JL, Weleber RG, Saperstein DA, Farrell DF, Stone EM (2009) Phenotypic variability due to a novel Glu292Lys variation in exon 8 of the BEST1 gene causing best macular dystrophy. Arch Ophthalmol 127:913-920

25. Mullins RF, Keuhn MH, Faidley EA, Syed NA, Stone EM (2007) Differential macular and peripheral expression of bestrophin in human eyes and its implication for best disease. Invest Ophthalmol Vis Sci 48:3372-3380

26. Cascavilla ML, Querques G, Stenirri S, Parodi MB, Querques L, Bandello F (2012) Unilateral vitelliform phenotype in autosomal recessive bestrophinopathy. Ophthalmic Res 48:146-150

27. Fung A, Yzer S, Allikmets R (2013) Clinical and genetic misdiagnosis of autosomal recessive bestrophinopathy. JAMA 131:1651 (Letter to the editor)

28. Pineiro-Gallego T, Alverez M, Pereiro I, Campos S, Sharon D, Schatz P et al (2011) Clinical evaluation of two consanguineous families with homozygous mutations in BEST1. Mol Vis 17:1607-1617

29. Pomares E, Bures-Jelstrup A, Ruiz-Nogales S, Corcostegui B, Gonzalez-Duatre R, Navarro R (2012) Nonsense-mediated decay as the molecular cause for autosomal recessive bestrophinopathy in two unrelated families. Invest Ophthalmol Vis Sci 53:532-537

30. Sharon D, Al-Hamdani S, Engelsberg K, Mizrahi-Meissonnier L, Obolensky B, Banin E et al (2014) Ocular phenotype analysis of a family with biallelic mutations in the BEST1 gene. Am J Ophthalmol 157:697-709

31. Preising MN, Pasquay C, Friedburg C, Bowl W, Jager M, Andrassi-Darida M et al (2012) Autosomal recessive betstrophinopathy (ARB): a clinical and molecular description of two patients at childhood. Klin Monbl Augenheilkd 229(10):1009-1017

32. Schatz P, Klar J, Andreasson S, Ponjavic V, Dahl N (2006) Variant phenotype of Best vitelliform macular dystrophy associated with compound heterozygous mutations in VMD2. Ophthalmol Genet 2:51-56

33. Bitner H, Mizrahi-Meissonnier L, Griefner G, Erdinest I, Sharon D, Banin E (2011) A homozygous frameshift mutation in BEST1 causes the classical form of Best disease in an autosomal recessive mode. Invest Ophthalmol Vis Sci 52:5332-5338

34. Low S, Davidson AE, Holder GE, Hogg CR, Bhattacharya SS, Black GC et al (2011) Autosomal dominant Best disease with an unusual electrooculographic light rise and risk of angle-closure glaucoma: a clinical and molecular genetic study. Mol Vis 17:2272-2282

35. Etter JR, Affel EL, Rhee DJ (2006) High prevalence of plateau iris configuration in family members of patients with plateau iris syndrome. J Glaucoma 15(5):394-398

36. Kramer F, White K, Pauleikhoff D, Gehrig A, Passmore L, Rivera A et al (2000) Mutations in the MD2 gene are associated with juvenile-onset vitelliform macular dystrophy (Best disease) and adult vitelliform macular dystrophy but not age-related macular degeneration. Eur J Hum Genet $8: 286-292$ 